\title{
Enquête clinique prospective portant sur l'extraction des troisièmes molaires sous anesthésie générale avec infiltration locale de lévobupivacaïne
}

\author{
Flora Thibaut $^{1,2}$, Fabrice Clipet ${ }_{1,2}^{1,2}$, Hubert Le Hétêt ${ }^{3}$, Valérie Bertaud ${ }^{1,2}$, \\ Nora Alno ${ }^{1,2}$, Patrick Limbour ${ }^{1,2}$ \\ ${ }^{1}$ Hôpital Pontchaillou, CHU, rue Henri Le Guilloux, 35000 Rennes, France \\ ${ }^{2}$ Pôle d'Odontologie et de Chirurgie buccale, CHU, 2 rue Henri Le Guilloux, 35033 Rennes, France \\ ${ }^{3}$ Hôpital privé de Sévigné, 8 rue Chêne Germain, 35510 Cesson-Sévigné, France
}

florathibaud@gmail.com

L'extractions des troisièmes molaires représente un acte fréquemment réalisé sous anesthésie générale qui peut générer des douleurs postopératoires (DPO) modérées à sévères durant la période postopératoire immédiate. La prévention des DPO représente un double enjeu : améliorer la qualité de vie des patients et diminuer la durée des hospitalisations lors d'interventions chirurgicales sous anesthésie générale. L'amélioration de la prise en charge des symptômes post-opératoires contribue ainsi au développement de la chirurgie ambulatoire et constitue un sujet d'actualité.

Dans ce contexte, une enquête clinique a été menée entre avril 2009 et février 2010 à l'Hôpital privé Sévigné dans le cadre d'un réseau ambulatoire. 76 patients ont été inclus dans cette enquête qui visait à analyser différents critères de morbidité postopératoire à la suite de l'extraction des troisièmes molaires sous anesthésie générale, associée à des infiltrations locales per-opératoires de lévobupivacaïne. Un suivi a été réalisé jusqu'au $7^{\text {ème }}$ jour post-opératoire, le critère principal étudié étant la DPO évaluée sur une échelle de 0 à 10.

Les patients ont décrit une intensité douloureuse située en moyenne entre 0,15 et 1,17 durant leur séjour à l'hôpital. Une majorité significative de patients (entre 75 et $80 \%$ ) n'a pas présenté de douleurs ou a décrit des DPO de faible intensité dans la période post-opératoire immédiate. Durant les premiers jours post-opératoires, une majorité significative de patients $(65,8 \%$ des patients à $\mathrm{J} 1$ et $72,4 \%$ à $\mathrm{J} 2)$ a présenté des DPO de faible intensité. Au cours du reste de la semaine suivant l'intervention, la plupart des patients ont décrit des DPO faibles $(52,6 \%)$ à modérées $(39,5 \%)$. Les autres suites ou complications post-opératoires observées concernaient une minorité de patients avec des taux variables.

Ce protocole, basé sur un principe d'analgésie préventive applicable en routine, est intéressant en terme de service médical rendu: le rapport entre efficacité et effets indésirables parait favorable, avec des DPO le plus souvent de faible intensité et une consommation d'antalgiques principalement représentées par des molécules de palier I ou de palier II (selon la classification de l'OMS).

En conséquence, l'utilisation de lévobupivacaïne lors de l'extraction des troisièmes molaires sous anesthésie générale en chirurgie ambulatoire semble présenter de multiples avantages : diminution de la morbidité post-opératoire en limitant notamment les DPO, avec en conséquence une réduction de la durée du séjour hospitalier, des arrêts de travail, des ré-hospitalisations et de la demande en soins primaires. Par ailleurs, la réalisation d'infiltrations anesthésiques locales associées à l'anesthésie générale permettrait de diminuer la consommation de morphiniques, réduisant ainsi leurs effets indésirables tels que les NVPO. Ce type de prise en charge génère ainsi des bénéfices cliniques et économiques et représente un réel enjeu de santé publique 